\title{
Comparative effects of Salvizan gel (containing Salvia officinalis extract) and triamcinolone acetonide gel on the duration and symptoms of oral lichenoid reactions
}

\author{
Parichehr Ghalayani $^{1}$, Fahimeh Pakravan², Fatemeh Abbasi \\ ${ }^{1}$ Dental Research Center, Department of Oral Medicine, Dental Research Institute, Isfahan University of Medical Sciences, Isfahan, Iran \\ ${ }^{2}$ Dental Implant Research Center, Department of Oral Medicine, Dental Research Institute, Isfahan University of Medical Sciences, Isfahan, Iran
}

\section{Abstract}

Objectives: Lichenoid reactions are disorders with similar clinical presentations. The current study was conducted to compare the improvement indices in the patients' symptoms by Salvizan and triamcinolone gels. Therefore, regardless of the cause, eliminating the pain and symptoms is one of the objectives of the treatment.

Methods: This study was designed as a randomized double-blind clinical trial. Diagnosis of patients was performed by clinical examinations and confirmed by histological evaluation. One group of patients was administered Salvizan and another group was administered triamcinolone. After washing their mouth, the patients administered the gel $(1 \mathrm{~cm})$ on the inflammatory mucosa three times a day for two weeks. The amelioration rate of the lesions in each visit was measured from according to Visual Analog Scale (VAS) on a scale of 0-10.

Results: The results of repeated measures ANCOVA showed a significant difference between groups in terms of the pain intensity before treatment $(p<0.001)$. Moreover, there was a significant difference between different times with regard to pain intensity $(p=0.004)$. To evaluate the clinical indices in the study groups, Mann-Whitney test was used and the results showed no significant difference for the extent of lesions before treatment ( $p=0.874)$, after one week $(p=0.503)$ and after two weeks $(p=0.669)$.
Conclusions: Salvizan could better reduce pain intensity than triamcinolone, but clinically no significant difference was reported between the drugs in terms of the extent of lesion.

Keywords: Lichenoid reaction, Gel, Scarlet sage, Triamcinolone

Citation: Ghalayani P, et al. (2022) Comparative effects of Salvizan gel (containing Salvia officinalis extract) and triamcinolone acetonide gel on the duration and symptoms of oral lichenoid reactions.

Dentistry 3000. 1:a001 doi:10.5195/d3000.2022.157

Received: March 3, 2021

Accepted: August 31, 2021

Published: March 2, 2022

Copyright: (C2022 Ghalayani P, et al. This is an open access article licensed under a Creative Commons Attribution Work 4.0 United States License.

Email:f.abbasi@dnt.mui.ac.ir

\section{INTRODUCTION}

\section{Lichenoid reactions are a set of} disorders with similar clinical presentations, but are created due to various reasons. These reactions are divided into such subtypes as lichen planus, druginduced lichen planus, contact lichenoid reactions and lichenoid reactions due to graft-versus-host disease (GVHD). Oral lichen planus
$(O L P)$ is a rather common and chronic oral disease that mostly affects the oral mucosa [1].

Oral lichen planus and lichenoid reactions are common oral mucosal diseases that are frequently encountered in the health centers. This mucocutaneous disease is characterized as desquamative gingivitis without symptoms and with Wickham striae or plaque and painful ulcers in oral mucosa. Although the primary cause of this disease is unknown, recent studies have shown different immune complexes along with responding cells for inflammatory structures and chronic lesions. Lichen planus is generally an immunologic disease that is microscopically similar to a hypersensitive reaction with $\mathrm{T}$-cell interference 
[2]. Factors such as diabetes, hepatitis $\mathrm{C}$, trauma and hypersensitivity to medications and metals can cause lichen planus [3]. Most of the patients with lichen planus are middleaged people. Women are also more affected by lichen planus than men. The prevalence of lichen planus has been reported to be $0.1-2.2 \%$.

OLP has different clinical features, but is mainly seen as reticular, erosive and bullous forms; the most common of which is reticular form [4]. Microscopically, OLP is characterized by hyperkeratosis, basal cell degeneration, basement membrane thickening and accumulated submucosal lymphocytes. The characteristics of oral lichenoid reactions include unilateral clinical presentation along with a significant number of plasma cells in lymphocytic community, accumulation of materials or cells in the tissues around the vessels, increase of colloid bodies and presence of acute inflammatory cells such as eosinophils and neutrophils $[5,6]$. OLP is induced as a result of such drug agents as thiazides, penicillamine, systemic diseases like chronic liver diseases, hepatitis $C$ virus, diabetes, and allergic reactions to metals such as amalgam, food supplies, flowers and Viral Hemorrhagic Disease
(VHD) $[3,7]$. The microscopic aspect of lichenoid reactions may have a similar presentation to OLP $[1,8]$.

Clinical manifestations of lichenoid reactions vary from white painless keratotic lesions to erosions and painful ulcers [9]. Although these lesions rarely threaten the patients' lives, the discomfort and pain associated with them can affect the normal function of mouth, increase the risk of infection and to some extent reduce the patients' quality of life. The main treatment for lichenoid reaction is administration of systemic and local corticosteroid [10]. Its local and potent type such as triamcinolone has increasingly been used for the treatment of lichenoid reaction [11].

Another treatment for lichen planus is medicinal plants [12-14]. Salvia officinalis is one of these plants whose extract is used in salvizan gel. Each $15 \mathrm{gr}$ of salvizan gel contains $28 \%$ hydroalcoholic extract. The leaves of Salvia officinalis are known to have antioxidant properties. These plants are used for local treatment of gingival inflammatory, microbial and painful complications and oral lesions [15]. This plant enjoys antimicrobial, antifungal and antivirus properties and is able to reduce salivary and sweat secretion. The leaves of this plant contain $3-8 \%$ catechin tannins, phenolic acids, monoterpenes, beta-thujone, thymol, cineol and water-soluble tannin [16].

This study was aimed to analyze the treatment effect of salvizan and triamcinolone gels among the patients with lichenoid reactions, provided that they would have a significant impact in the amelioration of mucosal ulcer and their complications would not be statistically significant.

\section{MATERIALS AND METHODS}

The study sample in this randomized double-blind clinical trial comprised of the patients with lichenoid reactions referring to the department of oral diseases at school of dentistry, Isfahan University of Medical Sciences in 2014. All the patients participated in the study voluntarily. This clinical trial study was registered in Iranian Registry of Clinical Trails (IRTC).

The inclusion criteria for the patients with lichenoid reaction included minimum age of 18 years, non-pregnant and nonbreastfeeding women and ability to remain during the study period. The patients with allergy to plant products and drug abuse and those who were reluctant to cooperate were excluded from the study. According to the Declaration of Helsinki, informed 
consent was taken from the patients.

In this study, the samples were selected through simple random sampling. The clinical criteria for diagnosis were based on the presence of ulcerative and bilateral lesions that were basically located in the cheeks and in some cases similar bilateral lesions that were at ventral surface, sides of the tongue and gums as desquamate gingivitis. Moreover, papules or reticular lines along the ulcerative strips were helpful in diagnosing most patients with lichenoid reactions. Finally, sampling was carried out for histopathological analysis for accurate diagnosis.

A total number of 30 samples in each group might yield a difference equal to $d=0.5$ between the mean scores at $\alpha=0.05$ up to $80 \%$. After taking the informed consent from the patients, they were divided into two groups; the first group underwent treatment with Salvizan gel and the second group was treated with triamcinolone gel.

The treatment was performed weekly and lasted for two weeks or until the lesions were treated (either of them). One group was administered Salvizan gel and the other group was administered triamcinolone gel. The drug was administered three times a day after eating the meal. The patients were provided with required trainings.

After eating food and washing their mouth, the patients rubbed the gel $(1 \mathrm{~cm})$ on the mucosa and massaged it. The recovery rate of lesions was measured in each visit according to the criteria presented in the study of Buajeeb et al, and the pain level was determined according to the Visual Analog Scale (VAS) from 0 to 10 . The pain level in patients was evaluated by a scaled ruler and VAS technique as well as the pain level perceived by the patient on the scale of $0-10$. In fact, VAS is a $10-\mathrm{cm}$ horizontal line on one end of which the term "no pain" and on the other end the term "severe pain" are printed. The patients were asked to draw a vertical line on the ruler based on the pain level they experienced at that moment. The extent of lesions is shown in Table 1 from 0 to 5 according to the parameters mentioned by Buajeeb [17].

Ulcer or burning, pain during eating and drinking, dry mouth, numbness after taking the studied drugs and use of products like caffeine, tobacco and alcohol were recorded in the patients' questionnaires (Table 2). The obtained data were analyzed by SPSS-21 software. Wilcoxon test was used to analyze the collected data in one group and compare it with another at different intervals and Mann-Whitney test was administered to compare two groups with each other. Survival analysis was performed as well.

Table1. The extent of lesions from 0 to 5 according to Buajeeb's criteria.

\begin{tabular}{cc}
\hline Grade & $\begin{array}{c}\text { The extent of lesions } \\
\text { according to Buajeeb's } \\
\text { criteria }\end{array}$ \\
\hline 0 & Normal mucosa \\
\hline 1 & $\begin{array}{c}\text { White poor and mild striae with } \\
\text { no erythematosus }\end{array}$ \\
\hline 2 & $\begin{array}{c}\text { White striae with atrophy less } \\
\text { than one square cm }\end{array}$ \\
\hline 3 & $\begin{array}{c}\text { White striae with atrophy more } \\
\text { than one square cm }\end{array}$ \\
\hline 4 & $\begin{array}{c}\text { White striae with erosion less } \\
\text { than one square cm }\end{array}$ \\
\hline 5 & $\begin{array}{c}\text { White striae with erosion more } \\
\text { than one square cm }\end{array}$ \\
\hline
\end{tabular}

\section{RESULTS}

Among the 30 patients under treatment with Salvizan gel, 8 patients were male and 22 patients were female, and among the 30 patients under treatment with triamcinolone gel, 10 patients were male and 20 of them were female. The age range of the patients was 20-60.

The results of repeated measures ANCOVA showed a statistically significant difference between Salvia officinalis and triamcinolone gels in terms of gender by 
controlling the effect of pain before treatment $(p=0.05)$. The pain intensity before treatment was reported to be significantly different in both groups $(p<0.001)$; however, no significant difference was observed between genders $(p=0.786)$. Also, VAS levels indicated a significant difference between different times $(p=0.004)$ (Figure 1).

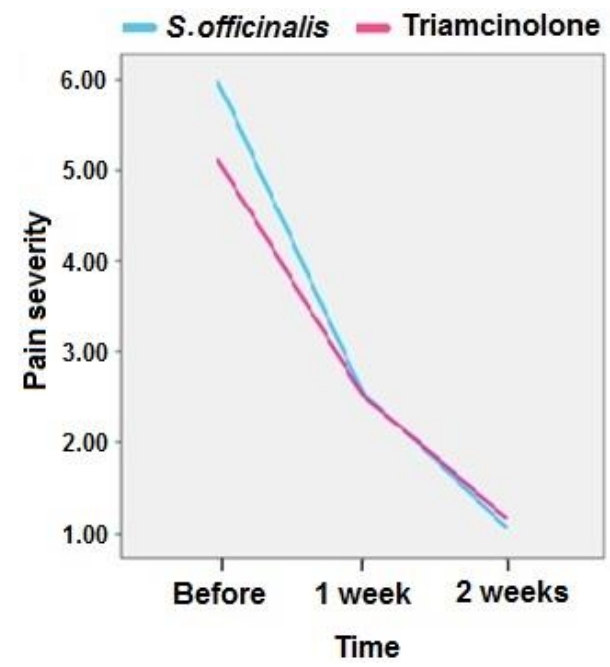

Figure 1. Standard deviation of pain level for both drugs at different times.

Following the ANCOVA test, paired sample t-test was run to compare VAS values at different times. The findings revealed a significant difference for Salvia officinalis between different times; before treatment and one week after treatment, before treatment and two weeks after treatment, and after one week and two weeks after treatment $(p<0.001)$. For triamcinolone, the same results were reported based on the paired sample t-test.

Table2 . Mean and standard deviation of pain intensity for both drugs at different times.

\begin{tabular}{ccccc}
\hline \multirow{2}{*}{ Drug } & Time & Mean & Number & SD \\
\hline \multirow{2}{*}{ Salvia officinalis } & Before treatment & 6.00 & 30 & 2.31 \\
& & & & \\
\cline { 2 - 5 } & One week after treatment & 2.56 & 30 & 1.75 \\
\cline { 2 - 5 } & Two weeks after treatment & 1.06 & 30 & 1.04 \\
\hline Triamcinolone & Before treatment & 5.13 & 30 & 2.17 \\
\cline { 2 - 5 } & One week after treatment & 2.53 & 30 & 1.75 \\
\cline { 2 - 5 } & Two weeks after treatment & 1.16 & 30 & 0.97 \\
& & & \\
\end{tabular}

To compare the clinical index of lichenoid reaction in the study groups, Mann-Whitney test was used for different times and the findings showed no significant difference between groups in terms of the extent of lesions before treatment $(p=0.874)$. This index was also not reported to be significantly different between groups one week after treatment $(p=0.503)$. For two weeks after treatment too, this index was not significantly different between groups $(p=0.669)$.

To compare the clinical index of Salvia officinalis and triamcinolone gels at different times, Friedman test was applied and the results indicated a significant difference between the three times in terms of the clinical index of both drugs $(p=0.001)$. Following Friedman test, Wilcoxon signed-rank test was used and the findings showed a significant difference between the three different times with regard to the clinical index of
Salvia officinalis and triamcinolone gels $(p<0.001)$.

\section{Discussion}

Lichenoid reactions are caused by numerous factors. Therefore, it seems necessary to make an effort to treat and eliminate the factors associated with the disease in order to prevent the complications resulting from it such as tooth loss due to health problems and possible malignant changes.

The treatment plan for lichenoid reactions is as follows: first, local corticosteroid is administered; second, the lesion is conservatively removed and the inducing factors are found using patch test, oral prophylaxis, restoration and regular polishing and using mouth wash. For more severe cases, chemotherapy and surgery are carried out; third, the patient undergoes control every three months for one year and checked again every two years. In 
the case of changes and recurrence of the disease, biopsy is performed again [18].

This study was carried out to evaluate the effect of salvizan and triamcinolone gels on 60 patients with lichenoid reactions. The findings indicated salvizan gel had a better effect than triamcinolone in terms of reducing the pain, although no significant difference was reported between them with regard to the extent of lesions. Based on the obtained results, it can be argued that salvizan, which is a medicinal plant, can be a substitute for triamcinolone, which is a chemical drug and has more side effects, or can be administered along with triamcinolone.

Medicinal plants have been used successfully to treat oral diseases [19-21]. As an example, Sookto et al showed that Saliva officinalis had anti-candida properties and prevented the connection of this fungus [22]. Moreover, Martin et al investigated the effect of oral patches containing licorice extract in the treatment of aphthous ulcers and reported positive effects in the size of the lesion and patient's pain [9]. Another nonpharmacologic and non-steroidal therapy used for the treatment of lichenoid diseases was the use of topical retinoic acid in comparison with topical fluocinolone acetonide which was investigated by Buajee et al. The authors concluded that topical fluocinolone acetonide was more effective to reduce the severity of lichen planus [17].

Moreover, Azizi et al compared the efficacy of Adcortyl lotion and topical tacrolimus and showed that topical tacrolimus could be an efficient and safe replacement for the treatment of lichen planus [23]. Furthermore, Bendas et al introduced hydroxychloroquine as a new and promising drug for the topical treatment of lichen planus for a short-term with few side effects [24].

The limitations of this study included lack of comprehensive control over the use of drugs and small sample size. Future studies are recommended to recruit larger study sample, compare different plant compounds, use plant complexes, and make use of topical cortones and plant medicines alternatively in order to obtain more accurate results.

\section{Conclusion}

Salvizan gel performed better than triamcinolone in decreasing pain intensity but clinically, no difference was reported between the two drugs with regard to the extent of lesion.

\section{REFERENCES}

1. Marx RE, Stern D. Oral and maxillofacial pathology. Chicago: Quintessence 2003;789-91.

2. Damm DD, Bouquot JE, Neville BW, Allen C. Oral and maxillofacial pathology. Chap. 2002;1:66.

3. Austyn JM, Wood K. Principles of cellular and molecular immunology Revista do Instituto de Medicina Tropical de São Paulo 1994;36(3):224-.

4. Edwards PC, Kelsch R. Oral lichen planus:clinical presentation and management. J Can Dent Assoc 2002;68(8):494-9

5. Boyd AS, Neldner KH. Lichen planus. J Am Acad Dermatol 1991;25(4):593-619.

6. Al-Hashimi I, Schifter $M$, Lockhart PB, Wray D, Brennan M, Migliorati CA, Axéll T, Bruce AJ, Carpenter W, Eisenberg E, Epstein JB. Oral lichen planus and oral lichenoid lesions: diagnostic and therapeutic considerations. Oral Surg Oral Med Oral Pathol Oral Radiol Endod 2007;103:25-e.

\section{McCreary CE, McCartan BE.} Clinical management of oral lichen planus. Br J Oral Maxillofac Surg 1999;37(5):338-43.

8. Carrozzo M, Gandolfo S. The management of oral lichen planus. Oral Dis 1999;5(3):196-205.

9. Martin MD, Sherman J, Van Der Ven P, Burgess J. A controlled trial of a dissolving oral patch 
containing glycyrrhiza (licorice) herbal extract for the treatment of aphthous ulcers. Gen Dent 2008;56:206-210.

10. Willershausen B, Gruber I, Hamm G. The influence of herbal ingredients on the plaque index and bleeding tendency of the gingiva. J Clin. Dent 1991;2(3):75-8.

11. Cherevatyi VS, Vashchenko TN, Shishkov GZ. Comparative evaluation of the antibacterial action of different extracts from Salvia officinalis. Rastitel'nye Resursy 1980;16(1):137-9.

12. Taheri E, Ghorbani S, Safi M, Sani NS, Amoodizaj FF, Heidari M, Chavoshi R, Hajazimian S, Isazadeh A, Heidari M. Inhibition of colorectal cancer cell line $\mathrm{CaCo}-2$ by essential oil of Eucalyptus camaldulensis through induction of apoptosis. Acta Med Iran. 2020;58(6):260-265.

13. Isazadeh A, Hajazimian $\mathrm{S}$, Mashayekhi M, Shadman B, Bedoustani AB, Safaei S, Chavoshi R, Shanehbandi D, Baradaran B, Nahaei M. Anti-cancer Effects of Probiotic Lactobacillus acidophilus for Colorectal Cancer Cell Line Caco-2 through Apoptosis Induction. Pharm Sci. 2021;27(2):368-373.

14. Jadid MF, Shademan B, Chavoshi R, Seyyedsani N, Aghaei E, Taheri E, Goleij P, Hajazimian S, Karamad V, Behroozi J, Sabet MN. Enhanced anticancer potency of hydroxytyrosol and curcumin by PLGA-PAA nano-encapsulation on PANC-1 pancreatic cancer cell line.
Environ Toxicol. 2021;36(6):10431051.

15. Willershausen B, Gruber I, Hamm G. The influence of herbal ingredients on the plaque index and bleeding tendency of the gingiva. J Clin Dent 1991;2(3):75-8.

16. Cherevatyî VS, Vashchenko

TN, Shishkov GZ. Comparative evaluation of the antibacterial action of different extracts from Salvia officinalis. Rastitel'nye Resursy 1980;16(1):137-9.

17. Buajeeb W, Kraivaphan P, Pobrurksa C. Efficacy of topical retinoic acid compared with topical fluocinolone acetonide in the treatment of oral lichen planus. Oral Surg Med Oral Patho Oral Radiol Endod 1997;83(1):21-5.

18. Kamath VV, Setlur K, Yerlagudda K. Oral lichenoid lesions-a review and update. Indian J Dermatol 2015;60(1):102.

19. Firouzi Amoodizaj F, Baghaeifar S, Taheri E, Farhoudi Sefidan Jadid M, Safi M, Seyyed Sani $\mathrm{N}$, Hajazimian S, Isazadeh A, Shanehbandi D. Enhanced anticancer potency of doxorubicin in combination with curcumin in gastric adenocarcinoma. J Biochem Mol Toxicol. 2020;34(6):e22486.

20. Mahdavi S, Kheyrollahi M, Sheikhloei $\mathrm{H}$, Isazadeh A. Antibacterial and Antioxidant Activities of Essential Oil on Food Borne Bacteria. Open Microbiol J. 2019;13(1):81-85.
21. Mahdavi S, Haj Azimian S, Isa Zadeh AR, Babash Pour M, Shishehgar R. Study of the antioxidant and antimicrobial effects of the ethanolic extract of Eucalyptus camaldulensis Dehnh against infectious bacteria isolated from clinical and animal sources. J Comparative Pathobiol. 2017;13(4):2063-70.

\section{Sookto T, Srithavaj T,} Thaweboon S, Thaweboon B, Shrestha B. In vitro effects of Salvia officinalis L. essential oil on Candida albicans. Asian Pac J Trop Biomed 2013;3(5):376-80.

23. Azizi A, Lawaf S. The comparison of efficacy of adcortyl ointment and topical tacrolimus in treatment of erosive oral lichen planus. J Dent Res Dent Clin Dent Prospects 2007;1(3):99-102.

24. Bendas ER, Abdullah $\mathrm{H}, \mathrm{El}-$ Komy MH, Kassem MA.

Hydroxychloroquine niosomes: a new trend in topical management of oral lichen planus. Int J Pharm 2013;458(2):287-95. 